rían en el tiempo y según el territorio vascular afectado. Objetivos. En este estudio nos propusimos estudiar cómo varía la sensibilidad y la relación con la clínica de la SPECT, el qEEG y la TC en las etapas aguda, subaguda y crónica, según el territorio vascular afectado. Asimismo, analizamos diferentes aspectos fisiopatológicos de las ECV isquémicas. Métodos. Se realizó un estudio con TC, qEEG, SPECT ${ }^{99 m} T c-H M P A O$ durante las etapas aguda (0-5 días), subaguda (6-15 días) y crónica (de 16 días a 1 año) a 36 pacientes. Resultados. La disminución ipsilateral del FSC dependió del tiempo ( $p=$ 0,0061 ), siendo poco frecuente durante las dos primeras semanas. El qEEG fue el estudio más sensible en la primera fase, su sensibilidad no estuvo relacionada con el territorio vascular afectado y sí dependió del tiempo $(p=0,0011)$, disminuyendo en la fase crónica. La actividad lenta habitualmente fue ipsilateral. La TC de cráneo resultó ser el estudio menos sensible. Conclusiones. Después de las 24 horas y hasta la segunda semana, habitualmente se produce un aumento del FSCr ipsilateral. La perfusión de lujo podría explicar el efecto fogging en la TC simple de cráneo. La actividad lenta del qEEG representa la alteración del metabolismo de oxígeno. La interpretación de la variación del FSC y del qEEG permite delimitar entre la perfusión de miseria de tipo oligoémica de la isquémica, asi como la hiperemia reactiva del aumento del FSC debido a la necrosis tisular [REV NEUROL 1998; 27: 213-23].

Palabras clave. ${ }^{99 m} T c-H M P A O$. Electroencefalografía computarizada. Flujo sanguíneo cerebral. Isquemia cerebral. SPECT. Trastornos cerebrovasculares. Tomografía computarizada. variam ao longo do tempo e de acordo com o território vascular envolvido. Objectivos. Neste estudo propusémo-nos estudar como varia a sensibilidade e a relação com a clínica da SPECT, do EEG e da TAC nas fases aguda, subaguda e crónica, de acordo com o território vascular envolvido, assim como analisar os diversos aspectos fisiopatológicos dos AVC isquémicos. Método. Realizou-se um estudo com TAC, EEG, SPECT ${ }^{99 m}$ Tc-HMPAO durante as fases aguda (0-5 dias), subaguda (6-15 dias) e crónica (de 16 dias a 1 ano) a 36 doentes. Resultados. A diminuição ipsilateral do FSC dependeu do tempo ( $p=0,0061)$, sendo pouco frequente durante as duas primeiras semanas. O EEG foi o estudo mais sensível na primeira fase, a sua sensibilidade não esteve relacionada com o território vascular envolvido mas antes dependeu do tempo $(p=0,0011)$, diminuindo na fase crónica. A actividade lenta foi geralmente ipsilateral. A TAC de crânio revelou-se como o estudo menos sensível. Conclusões. Despois das 24 horas e até à segunda semana, há geralmente um aumento do FSCr ipsilateral. A perfusão de luxo poderia explicar o efeito fogging na TAC simples de crânio. A actividade lenta do EEG representa a alteração do metabolismo do oxigénio. A interpretação da variação do FSC e do EEG permite delimitar entre a baixa perfusão de tipo oligoémica da isquémica, assim como a hiperémia reactiva do aumento do FSC devido à necrose tecidular [REVNEUROL 1998; 27: 213-23].

Palavras chave. ${ }^{99 m}$ TC-HMPAO. Acidentes vasculares cerebrais. Electroencefalografía computarizada. Fluxo sanguíneo cerebral. Isquémia cerebral. SPECT. Tomografía computarizada.

\title{
Afectación parenquimatosa del sistema nervioso central en la enfermedad de Behçet
}

\author{
A. Martínez-Yélamos, A. Vidaller ${ }^{\text {a }}$, F. Mitjavila ${ }^{\text {a }}$, R. Pujol ${ }^{\text {a }}$, M. Jato, F. Rubio
}

\section{INVOLVEMENT OF THE PARENCHYMA OF THE CENTRAL NERVOUS SYSTEM IN BEHCET DISEASE}

\begin{abstract}
Summary. Introduction. Behçet disease is a systemic form of vasculitis which presents with neurological symptoms with a frequency varying between 16 and $40 \%$. Involvement of the parenchyma has been found to worsen the prognosis in patients with neuroBehçet $(N B)$. Objective. To review the clinical features and course of patients with NB involving the parenchyma of the central nervous system (CNS). Clinical cases. Seven patients with Behçet disease and neurological localizing signs were seen in our hospital between 1989 and 1996. The initial diagnosis was of ischemic ictus in five of the seven patients. Both neuroimaging studies and investigation of the cerebrospinal fluid were always pathological in all cases. Vascular studies (arteriography and echoDoppler of the supra-aortic trunks) were normal. One patient died. Four patients had serious sequelae following treatment. Conclusion. NB should be included in the differential diagnosis of ictus. Involvement of the parenchyma of the CNS was accompanied by lymphocytic meningitis, perhaps also leading to a worse functional prognosis [REV NEUROL 1998; 27: 223-5]. Key words. Azathioprine. Behçet. Lymphocytic meningitis. Stroke.
\end{abstract}

\section{INTRODUCCIÓN}

La enfermedad de Behçet es una vasculitis sistémica que se caracteriza, además de la clásica tríada de aftas orales recidivantes, aftas genitales y lesiones oculares, por otras manifestaciones clínicas como la afectación vascular, articular, intestinal y neurológica; esta última es relativamente frecuente (entre el 16 y el 40\%) [1] y se denomina entonces neurobehçet (NB). Entre los cuadros neurológicos que se presentan en esta enfermedad destacan la trombosis venosa cerebral [2], hipertensión intracraneal aislada [3], meningitis o meningoencefalitis, infartos isquémicos [4,5] y polirradiculoneuritis. La meningoencefalitis constituye la forma más frecuente de presentación y es rara la afectación del sistema

Recibido: 29.11.97. Recibido en versión revisada: 10.01.98. Aceptado: 12.02.98. Servicio de Neurología. ${ }^{a}$ Servicio de Medicina Interna. CSU de Bellvitge. L'Hospitalet de Ll., Barcelona, España. nervioso periférico [1]. El objetivo del presente trabajo es revisar la clínica y la evolución de los pacientes con afectación parenquimatosa del sistema nervioso central y enfermedad de Behçet diagnosticados en la CSU de Bellvitge.

\section{CASOS CLÍNICOS}

Entre 1989 y 1996 se diagnosticaron siete enfermos de NB con afectación parenquimatosa de acuerdo con los criterios de O'Duffy [6]. Se analiza, a continuación, la clínica, tratamiento y evolución de estos pacientes.

La edad media fue de 38 años (rango 27-56). El sexo predominante en nuestra serie fue el masculino ( 6 varones $/ 1$ mujer). Todos los pacientes presentaban o habían presentado aftas orales recurrentes dolorosas, el $57 \%$ aftas genitales, el $57 \%$ patergia o foliculitis, el $71 \%$ artritis o artralgias y el

Correspondencia: Dr. Francisco Rubio Borrego. Servicio de Neurología. CSU de Bellvitge. E-08907 L'Hospitalet de Ll., Barcelona.

(C) 1998, REVISTA DE NEUROLOGÍA 


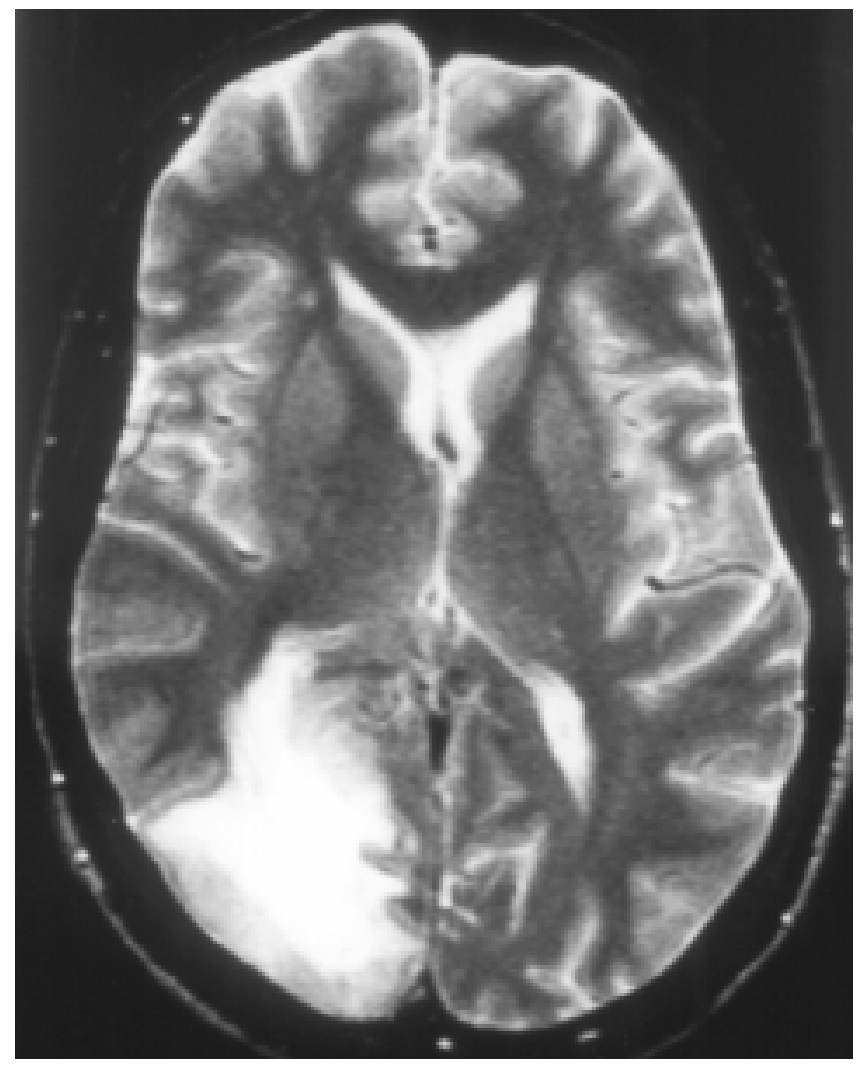

Figura 1 RMN de un paciente con enfermedad de Behçet que presentaba clínica de meningoencefalitis con hemianopsia homónima izquierda. Se observa hiperintensidad en $\mathrm{T}_{2}$ de predominio en sustancia blanca de localización occipital derecha.

43\% uveítis (Tabla I). La aparición de focalidad neurológica fue el motivo de consulta en todos los casos y en tres pacientes significó la forma de debut de la enfermedad.

El diagnóstico inicial fue de ictus isquémico en cinco de los siete pacientes y cuatro enfermos padecían, además, cefalea. La focalidad neurológica apreciada con mayor frecuencia fue la presencia de un síndrome piramidal (Tabla I). Los estudios por neuroimagen resultaron patológicos en todos los casos (Fig. 1). Dos pacientes, cuya TAC craneal fue normal, presentaron alteraciones en la resonancia magnética nuclear (RMN); uno de estos enfermos presentaba una meningomielitis que se detectó en la RMN medular. La arteriografía craneal y la eco-Doppler de troncos supraórticos (TSAo) realizadas a tres y cinco pacientes, respectivamente, no mostró alteraciones relacionadas con la focalidad neurológica en ninguno de ellos (Tabla II). La punción lumbar resultó patológica en todos los casos en que fue realizada (6/6). La presencia de pleocitosis fue el hallazgo más frecuente. Las lesiones se localizaron por neuroimagen en ganglios basales y cápsula interna ( 3 pacientes), tronco (2), sustancia blanca occipital (1) y un caso de meningomielitis. Un enfermo, con lesión en tronco que requirió intubación orotraqueal, fue éxitus letalis por hemorragia digestiva alta, sin que fuese posible realizar el estudio necrópsico.

Los pacientes fueron tratados inicialmente con prednisona en dosis altas $(1 \mathrm{mg} / \mathrm{kg} / \mathrm{d}$ áa). Se eligió la azatioprina como tratamiento de elección, en el caso de no haber obtenido respuesta a los corticosteroides, por ser el único fármaco con estudio controlado en el tratamiento de la enfermedad de Behçet [7]. Todos los pacientes fueron tratados inicialmente en régimen hospitalario y controlados posteriormente de forma ambulatoria cada tres meses. Cuatro enfermos respondieron inicialmente a la prednisona. Actualmente dos enfermos se encuentran libres de síntomas neurológicos y los otros dos están bajo tratamiento corticoideo presentando un grado de incapacidad ligero según la escala de Rankin. A los dos pacientes que no respondieron a la prednisona se les añadió azatioprina (ambos la toman en la actualidad en dosis bajas); son los únicos enfermos que se encuentran con un grado de incapacidad entre moderado o grave (Fig. 2).

\section{N. de pacientes}

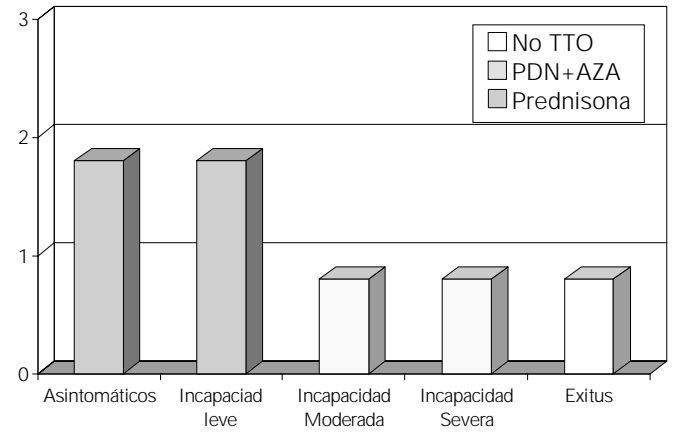

Figura 2. Situación funcional de los pacientes seguidos (grados de incapacidad según la escala de Rankin) y tratamiento que siguen en la actualidad. Los pacientes que precisaron y precisan un tratamiento con inmunosupresores para controlar los brotes neurológicos se encuentran con mayor incapacidad.

Tabla I. Distribución de las manifestaciones sistémicas y neurológicas de los pacientes.

\begin{tabular}{lcc}
\hline Clínica & N.\% total & $\%$ \\
\hline Aftas orales & $7 / 7$ & 100 \\
\hline Aftas genitales & $4 / 7$ & 57 \\
\hline Artritis & $5 / 7$ & 71,5 \\
\hline Patergia/Foliculitis & $4 / 7$ & 57 \\
\hline Uveítis & $3 / 7$ & 43 \\
\hline Clínica neurológica & $7 / 7$ & 100 \\
\hline Signos piramidales & & N.o de pacientes \\
\hline Déficit sensitivo & & 5 \\
\hline Hemianopsia & & 3 \\
\hline Síndrome cerebeloso & & 1 \\
\hline Aftas pares craneales & & 1 \\
\hline Coma & & 1 \\
\hline
\end{tabular}

\section{DISCUSIÓN}

Describimos siete pacientes con enfermedad de Behçet y afectación neurológica (neurobehçet) en los que se demostró afectación parenquimatosa. La aparición de accidentes vasculares cerebrales isquémicos se describe hasta en un $25 \%$ de enfermos con NB [4]. Se ha descrito en la literatura la existencia de accidentes isquémicos transitorios, infartos profundos [8] y extensos [9]. Por otra parte, raramente se ha demostrado la oclusión de arterias cerebrales [10]. Cinco de nuestros pacientes fueron diagnosticados inicialmente de ictus isquémico por las características clínicas del cuadro neurológico inicial, no mostrando el estudio vascular alteraciones relacionadas con la focalidad neurológica. Es discutible si la clínica neurológica parenquimatosa es debida a necrosis vascular como resultado de la oclusión inflamatoria de pequeñas arterias cerebrales o es resultado de la inflamación, la desmielinización y la gliosis [11]. Sin embargo, parece evidente que la en- 
Tabla II. Resultado de las diferentes exploraciones complementarias. La resonancia magnética (RM) fue más sensible que la tomografía computarizada (TAC). La punción lumbar resultó siempre patológica y el estudio vascular fue normal en todos los casos.

\begin{tabular}{lcc}
\hline Exploraciones & Normal & Patológica \\
\hline TAC & 2 & 5 \\
\hline RMN & 1 (RMN medular anormal) & 5 \\
\hline Eco-Doppler TSAo & 5 & 0 \\
\hline Angiografía & 3 & 0 \\
\hline Punción lumbar & 0 & 6 \\
\hline
\end{tabular}

fermedad de Behçet tiene que formar parte del diagnóstico diferencial del ictus isquémico, especialmente en pacientes jóvenes.

Todos nuestros pacientes tenían focalidad neurológica concordante con lesiones demostradas en los estudios de neuroimagen. La RMN se mostró más sensible que la TAC en la detección de dichas lesiones [12]. Al mismo tiempo, todos los pacientes mostraron alteraciones en el líquido cefalorraquídeo concordan- tes con meningitis linfocitaria $[7,13]$. El $66 \%$ de nuestros pacientes presentan algún tipo de déficit neurológico residual concordante con la persistencia de lesiones observada en la RMN. El pobre pronóstico funcional de nuestros enfermos se puede explicar por el hecho de que todos tenían afectación parenquimatosa del sistema nervioso central. Otros autores ya han apuntado la posible existencia de dos grupos de pacientes dentro de los enfermos con NB, unos con afectación únicamente vascular (trombosis venosas) y otros con afectación parenquimatosa del sistema nervioso central; estos últimos son los que tendrían un peor pronóstico [3].

\section{CONCLUSIÓN}

1. El NB ha de formar parte del diagnóstico diferencial del ictus isquémico, siendo la historia clínica la base para la sospecha diagnóstica en estos pacientes; 2. La RMN es útil cuando la TAC es normal; 3. La afectación parenquimatosa se acompaña en todos nuestros casos de meningitis linfocitaria, y 4. El estudio vascular no muestra alteraciones en casos de NB con afectación parenquimatosa, y dicha afectación puede condicionar un peor pronóstico funcional.
1. Benamour S, Zeroual B, Bennis R, Amraoui A, Bettal S. Maladie de Behcet. 316 cas. Presse Med 1990; 19: 1485-9.

2. Wechsler B, Vidailhet M, Piette JC, Bousser MG, Dell Isola B, Blétry $\mathrm{O}$, et al. Cerebral venous thrombosis in Behçet's disease: clinical study and long-term follow-up of 25 cases. Neurology 1992; 42: 614-8.

3. Akman-Demir G, et al. Intracranial hypertension in Behçet's disease. Eur J Neurol 1996; 3: 66-70.

4. O'Duffy JD. Behçet's syndrome. NEJM 1990; 322: 326-7.

5. Vidaller A, Carratala J, Moreno R, Arbizu T, Rubio F. Magnetic resonance imaging in neuro-Behçet disease. Br J Rheumatol 1988; 27 : 79-80.

6. O'Duffy JD, Goldstein NP. Neurologic involvement in seven patients with Behçet's disease. Am J Med 1976; 61: 170-8.

7. Yazici H, Pazrli H, Barnes CG, Tüzün Y, Özyazgan Y, Silman A, et al. A controlled trial of azathioprine in Behçet's syndrome. NEJM 1990; 332: 281-5.

8. Bertol V, Ara JR, Oliveros A, Gutiérrez AI, Samperiz P, Gros B, Al-

\section{AFECTACIÓN PARENQUIMATOSA DEL SISTEMA NERVIOSO CENTRAL EN LA ENFERMEDAD DE BEHÇET}

Resumen. Introducción. La enfermedad de Behçet es una vasculitis sistémica que presenta clínica neurológica con una frecuencia que varía entre el 16 y el 40\%. Se ha relacionado la afectación parenquimatosa con un peor pronóstico en los pacientes con neurobehçet (NB). Objetivo. Revisar la clínica y evolución de los pacientes con NB y afectación parenquimatosa del sistema nervioso central $(S N C)$. Casos clínicos. Siete pacientes con enfermedad de Behçet y focalidad neurologica fueron atendidos en nuestro centro entre 1989 y 1996. El diagnóstico inicial fue de ictus isquémico en cinco de los siete pacientes. Tanto los estudios de neuroimagen como los del líquido cefalorraquídeo resultaron siempre patológicos. Los estudios vasculares (arteriografía y eco-Doppler de troncos supraórticos) fueron normales. Un enfermo fue éxitus letalis. Cuatro pacientes presentaron secuelas tras el tratamiento. Conclusión. El $N B$ ha de formar parte del diagnóstico diferencial del ictus. La afectación parenquimatosa del SNC se acompaña de meningitis linfocitaria y puede, además, condicionar un peor pronóstico funcional [REV NEUROL 1998; 27: 223-5].

Palabras clave. Azatioprina. Behçet. Ictus. Meningitis linfocitaria. márcegui C. Neuro-Behçet. Estudio clínico de 9 pacientes. Neurología 1992; 7: 20-4.

9. Villagra Cocco PV, Pascual Gamero L, Villagra Cocco A, Yusta Izquierdo A. Síndrome de Balint como manifestación de la enfermedad de Behçet. Rev Esp Neurol 1992; 191: 339-40.

10. Iragui VJ, Marari E. Beçet syndrome presenting as cerebrovascular disease. J Neurol Neurosurg Psychiatry 1986; 49: 838-40.

11. Martínez JM, Barraquer-Bordas Ll, Ferrer I, Escartin AE, Rosich M, Barraquer-Feu MLl. Étude anatomo-clinique d'un syndrome de Behçet avec atteinte du système nerveux central. Rev Neurol (Paris) 1988; 144: 130-5.

12. Wechsler B, Dell Isola B, Vidailhet M, Dormont D, Piette JC, Blétry $\mathrm{O}$, et al. MRI in 21 patients with Behçet's disease and neurological involvement: prospective study with clinical correlation. J Neurol Neurosurg Psychiatry 1993; 56: 793-8.

13. Serdaroglu P, Yazici H, Ozdemir C, Yurdakul S, Bahar S, Aktin E. Neurologic involvement in Behçet's syndrome. A prospective study. Arch Neurol 1989; 46: 265-9.

\section{ENVOLVIMENTO PARENQUIMATOSO DO SISTEMA NERVOSO CENTRAL NA DOENÇA DE BEHÇET}

Resumo. Introdução. A doença de Behçet é uma vasculite sistémica que apresenta sinais e sintomas neurológicos com uma frequência que varia entre 16 e $40 \%$. Relacionou-se a existência de envolvimento parenquimatoso com um pior prognóstico nos doentes com neuroBehçet (NB). Objectivo. Rever a apresentação clínica e a evolução dos doentes com NB e envolvimento parenquimatoso do sistema nervoso central (SNC). Casos clínicos. Foram observados no nosso centro sete doentes com doença de Behçet e apresentação neurológica focal entre 1989 e 1996. O diagnóstico inicial foi de ictus isquémico em cinco dos sete doentes. Tanto os estudos imagiológicos cerebrais como os do líquido cefalorraquidiano apresentaram sempre alterações patológicas. Os estudos vasculares (arteriografía e eco-Doppler dos troncos supra-aórticos) foram normais. Um doente teve éxitus letalis. Quatro doentes apresentaram sequelas após o tratamento. Conclusão. $O N B$ deverá fazer parte do diagnóstico diferencial da isquémia cerebral. O envolvimento parenquimatoso do SNC acompanha-se de meningite linfocitária, podendo, além do mais, condicionar um pior prognóstico funcional [REV NEUROL 1998; 27: 223-5].

Palavras chave. Azatioprina. Behçet. Isquémia cerebral. Meningite linfocitária. 